\title{
Ausbau der allgemeinen Palliative Care in der Grundversorgung
}

\section{Marianne Lang Meier}

Dr. med., frühere Präsidentin der Arbeitsgruppe Qualität von palliative ch

Die Schweizerische Gesellschaft für Palliative Medizin, Pflege und Begleitung, palliative ch, hat Empfehlungen für die allgemeine Palliative Care veröffentlicht [1, 2]. Diese Empfehlungen richten sich an die Leistungserbringer der ambulanten und stationären medizinischen Grundversorgung. Sie können hilfreich sein bei Aufund Ausbau von lokalen oder regionalen Palliative-Care-Netzwerken.

\section{Entstehung, Sinn und Nutzen der Empfehlungen}

Mit der Nationalen Strategie Palliative Care 2010-2012 [3] haben Bund (d.h. das Bundesamt für Gesundheitswesen BAG) und Kantone (die GesundheitsdirektorenKonferenz GDK) einen entscheidenden Anstoss zur Förderung der Palliative Care in der Schweiz gegeben. In dieser ersten Strategiephase (2010-2012) wurde der Schwerpunkt auf die spezialisierte Palliative Care gelegt.

\section{Ein wichtiges Anliegen war und ist die Gewährleistung der Qualität der palliativen Versorgung.}

In der Weiterführung der Nationalen Strategie Palliative Care 2013-2015 [4] wurde als Folge und Ergebnis der ersten Phase explizit das Gewicht auf die Allgemeine Palliative Care, also die Palliative Care in der medizinischen Grundversorgung, gelegt.

Ein wichtiges Anliegen der Nationalen Strategie und der nachfolgenden Empfehlungen für die Umsetzung war und ist die Gewährleistung der Qualität der palliativen Versorgung.

Die Arbeitsgruppe Qualität, eine der Arbeitsgruppen von palliative ch, befasst sich seit langer Zeit mit der Erarbeitung von Qualitätskriterien für die spezialisierte Palliative Care für den stationären und den ambulanten Bereich.

Diese Qualitätskriterienlisten sind das Instrument für Audits, d.h. Qualitätsüberprüfungen, in den spezialisierten ambulanten und stationären Strukturen und Institutionen, auch in spezialisierten Langzeitpflege- institutionen. Diese Audits werden von geschulten Peers unter der Leitung von Auditierungs- bzw. Zertifizierunsgsfirmen durchgeführt und berechtigen die Institutionen, falls sie bestehen, das Label für spezialisierte Palliative Care für eine definierte Zeit zu führen. In der medizinischen Grundversorgung ist eine systematische Qualitätsüberprüfung, wie oben beschrieben, nicht möglich. $\mathrm{Zu}$ vielgestaltig sind die Settings der selbständig arbeitenden Fachleute und der Institutionen, die sich um die Patienten in der Grundversorgung kümmern, als dass sie miteinander vergleichbar wären und einen starren Katalog von Kriterien erfüllen könnten. Es würde sich auch ein riesiges personelles und logistisches Problem ergeben, flächendeckend Qualitätskontrollen durchzuführen.

Um dieser Andersartigkeit Rechnung zu tragen, aber den Grundversorgern doch auch ein Instrument für Aufbau und Überprüfung ihrer Arbeit in Palliative Care in die Hand zu geben, ist die Arbeitsgruppe Qualität einen anderen Weg gegangen. Sie hat in Zusammenarbeit mit einer Gruppe von Fachleuten aus der medizinischen Grundversorgung elf Empfehlungen formuliert als Handlungsanweisungen. Diese Handlungsanweisungen sind gedacht als praxisorientiertes Instrument, welches die Umsetzung der von BAG, GDK und palliative ch erarbeiteten Vorschläge und Forderungen zum Ausbau von Palliative Care erleichtern und fördern soll.

\section{Die elf Empfehlungen}

Jede Empfehlung wird durch einen kürzeren oder längeren Text vertieft und gelegentlich, wo sinnvoll, werden links direkt im Text angegeben. 
Im Folgenden werden die Titel der elf Empfehlungen aufgelistet und mit einem kurzen Ausschnitt aus den jeweiligen Erklärungen vertieft:

\section{Ein Konzept erarbeiten}

«... Dieser Prozess löst die Reflexion über die eigene Haltung, das eigene Handeln und über die Zusammenarbeit mit anderen Fachpersonen aus ..."

2. Palliative Situationen erkennen «Es ist grundlegend, Situationen zu erkennen, in denen Palliative Care nötig oder wünschenswert wäre, und dies frühzeitig und feinfühlig mit Patientin oder Patient und Angehörigen zu besprechen ...»

3. Bedürfnisse erfassen

«Der Wille und die Wünsche der Patientin oder des Patienten und seiner Angehörigen bezüglich Palliative-Care-Betreuung sind zu erfassen, über Erwartungen und Befürchtungen zu sprechen ...»

4. Über das Sterben reden

«... Um über Leben, Sterben und Tod sprechen zu können und die kommunikativen Fähigkeiten zu stärken, werden Fallbesprechungen, Debriefings, Supervisionen in den Teams, zugezogene Fachleute und regionale Netzwerke genutzt.»

5. Vernetzt und interprofessionell arbeiten

«Palliative Care wird idealerweise in einem interprofessionellen Team oder Netzwerk erbracht. Fachpersonen der universitären und nicht-universitären Gesundheitsberufe, der Sozial- und Psychologieberufe, der Seelsorge, weiterer Berufe, aber auch Freiwillige können Teil des Netzwerkes sein ...»

6. Zum Symptommanagement beitragen «... Alle an der Betreuung Beteiligten unterstützen die Erfassung, die Behandlung und die Linderung von Symptomen ...»

7. Zu optimaler End-of-Life Care beitragen

«Die Fachpersonen der medizinischen Grundversorgung sind befähigt, in der Kontinuität der Palliative Care zu guter End-of-Life Care kompetent beizutragen. Dank Teamarbeit, Vernetzung und guter Kommunikation erhalten die Patientin oder der Patient und Angehörige in dieser schwierigen Phase die nötige Unterstützung ...»

8. Angehörige einbeziehen und betreuen

«Die Angehörigen sind sehr wichtig in der Betreuung und sollten gemäss ihren Bedürfnissen angeleitet, einbezogen, unterstützt und entlastet werden ...»

9. Patientenrechte und Kindes- und Erwachsenenschutzgesetz kennen

«Die Fachpersonen kennen die Patientenrechte und die Kerninhalte des seit 2013 in Kraft getretenen Kindes- und Erwachsenenschutz-Gesetzes (KESG).
Sie sind vertraut mit Patientenverfügungen und Vorsorgeaufträgen ...»

\section{Sich mit ethischen Fragen auseinandersetzen}

«Die Fachpersonen und das Team setzen sich mit ethischen Fragestellungen und gemeinsamer Entscheidungsfindung auseinander ...»

11. Bedürfnisgerechte Aus-, Weiter- und Fortbildung absolvieren

«... Das Angebot von lokalen, regionalen und überregionalen Veranstaltungen erlaubt, Kenntnisse und Kompetenzen zu vertiefen, Haltungen $\mathrm{zu}$ hinterfragen und zu stärken.»

Die Empfehlungen sind nach ihrer Erarbeitung einer breiten Vernehmlassung bei den Standesorganisationen, Berufsverbänden, Interessengruppen usw. unterzogen worden und nach entsprechenden Korrekturen 2018 veröffentlicht worden. Nebst Einleitung, Präambel und Anhang enthält die Broschüre als zentrales Element die elf Empfehlungen. Sie sind eher knapp formuliert, geben aber dabei konkrete Hinweise und Anleitungen für den Auf- und Ausbau von PalliativeCare-Angeboten. Nach Meinung der Autoren sind sie so allgemein gehalten, dass sie geeignet sind für die Anwendung im ganzen Spektrum von Fachpersonen und Institutionen: Pflegefachleute, Spitex-Dienste, Spitalärzte, Hausärzte, Gruppenpraxen, Physiotherapeuten, Ergotherapeuten, Seelsorgende, Psychologen, Sozialarbeiter, Ernährungsberater, Kunst-, Musik-, Mal- und Tanztherapeuten, komplementärmedizinische Therapeuten und andere.

Die Empfehlungen sind ebenfalls gedacht für den stationären Bereich der medizinischen Grundversorgung. Auch Spitäler und Kliniken, Alters- und Pflegeheime, Institutionen für Menschen mit Behinderungen, Pflegeabteilungen von Gefängnissen sollten damit über ein einfach zu handhabendes Instrument für die Einführung oder Verbesserung der Palliative Care verfügen.

Die Empfehlungen sind aufgeschaltet auf der Website palliative.ch [2] und erhältlich als gedruckte Broschüre [1].

\section{Entstehung und Entwicklung der Palliative Care}

Ein kurzer historischer Rückblick zeigt uns, dass Palliative Care in den sechziger Jahren des letzten Jahrhunderts in England entstanden ist. Sie hat sich als Bewegung international im Bereich der Onkologie weiterentwickelt und ist in wenigen Jahren zu einem eigenen Fach geworden. Sie findet immer mehr Eingang in andere medizinische Spezialitäten (Neurologie, Geria- 
trie, Pneumologie, Pädiatrie, um nur ein paar zu nennen) und wird gemäss deren Besonderheiten angepasst und organisiert.

Auch in der Schweiz haben sich seit den achtziger Jahren des letzten Jahrhunderts in verschiedenen Kantonen und Regionen Gruppen, Vereine und Organisationen im Bereich Palliative Care gebildet, meist in Verbindung mit der Onkologie. Die Schweizerische Gesellschaft für Palliative Medizin, Pflege und Begleitung wurde 1988 gegründet und heisst palliative $c h$. Sie ist eine multiprofessionelle Fachgesellschaft mit 14 kantonalen bzw. regionalen Sektionen und verschiedenen Fach- und Arbeitsgruppen sowie Task Forces und zählt aktuell circa 3000 Mitglieder.

\section{Nationale Strategie Palliative Care Schweiz}

Wie bereits oben erläutert, hat sich seitens der Politik vieles getan in den letzten Jahren. Mit der ersten Nationalen Strategie Palliative Care 2010-2012 [3] haben Bund und Kantone einen entscheidenden Anstoss zur Förderung der Palliative Care in der Schweiz gegeben. Schwerpunkt war die spezialisierte Palliative Care. In der Weiterführung der Nationalen Strategie 2013-2015 ging es vor allem um die Palliative Care in der medizinischen Grundversorgung. Die drei Akteure Bund (BAG), Kantone (GDK) und palliative ch haben im

\section{Das Wichtigste in Kürze}

- In der medizinischen Grundversorgung ist eine systematische Qualitätsüberprüfung der Palliative Care nicht möglich - zu vielgestaltig sind die Settings der selbständig arbeitenden Fachleute und Institutionen.

- Die Arbeitsgruppe Qualität hat deshalb in Zusammenarbeit mit einer Gruppe von Fachleuten aus der medizinischen Grundversorgung elf Empfehlungen als Handlungsanweisungen formuliert.

palliative ch

Bubenbergplatz 11

CH-3011 Bern

info[at]palliative.ch

www.palliative.ch

Marianne Lang Meier

malamei[at]bluewin.ch
- Die Empfehlungen sind nach ihrer Erarbeitung einer breiten Vernehmlassung bei den Standesorganisationen, Berufsverbänden, Interessengruppen usw. unterzogen worden und nach entsprechenden Korrekturen 2018 veröffentlicht worden.

- Die Empfehlungen sind aufgeschaltet auf derWebsite palliative.ch [2] und erhältlich als gedruckte Broschüre [1].
August 2015 ein Dokument [5] herausgegeben, welches die Gründe für diesen Handlungsbedarf ausführlich darlegt und Wege aufzeigt, wie vorgegangen werden soll. Das Dokument ist umfassend und erläutert auf 41 Seiten die folgenden Themen der allgemeinen Palliative Care: Zielgruppen, Leistungen, Strukturen und Kompetenzen.

Die «Empfehlungen» sind ein Beitrag zu den politischen Bemühungen für die Verbreitung der Palliative Care in der Grundversorgung und erlauben dank ihrer Kürze einen gezielten Einstieg in die Palliative Care. Sie sollen als Orientierung und auch als Checkliste dienen. Wir hoffen, dass sie breite Anwendung finden werden.

\section{Disclosure statement}

Die Autorin erklärt keine Interessenverbindungen in Zusammenhang mit dem vorliegenden Manuskript.

\section{Literatur}

1 Allgemeine Palliative Care. Empfehlungen für die Fachpersonen und Leistungserbringer der ambulanten und stationären medizinischen Grundversorgung, palliative ch 2018.

2 https://www.palliative.ch/fileadmin/user_upload/palliative/ fachwelt/C Fachgesellschaft/Arbeitsgruppen/Qualitaet/Broschuere_Allg_PalliativeCare_Empf_fuer_Fachpersonen_de_RL3.pdf

3 Nationale Strategie Palliative Care 2010-2012, BAG und GDK, 2009.

4 Nationale Strategie Palliative Care 2013-2015, BAG und GDK, 2012.

5 Bundesamt für Gesundheitswesen BAG, Schweizerische Konferenz der Gesundheitsdirektorinnen und -direktoren GDK und palliative ch (2015): Allgemeine Palliative Care. Empfehlungen und Instrumente für die Umsetzung. Bern.

\section{L'essentiel en bref}

- Un contrôle systématique de la qualité des soins palliatifs n'est pas possible dans les soins de santé primaires, les cadres dans lesquels travaillent les professionnels indépendants et les institutions sont par trop différents.

- Conjointement avec un groupe de spécialistes des soins de santé primaires, le groupe de travail Qualité a donc formulé onze recommandations sous forme d'instructions d'action.

- Une fois élaborées, ces recommandations ont été soumises aux organisations et associations professionnelles, aux groupes d'intérêt, etc. dans le cadre d'une vaste consultation, puis ont été publiées en 2018 après les corrections appropriées.

- Les recommandations sont disponibles en ligne sur le site Web palliative.ch [2] et sous forme de brochure papier [1] 